\title{
A Method for Estimating Hunger Degree based on Meal and Exercise Logs
}

\author{
Isamu Sugita, Morihiko Tamai, Yutaka Arakawa, Keiichi Yasumoto \\ Graduate School of Information Science, Nara Institute of Science and Technology \\ Ikoma, Nara 630-0192, Japan Email: \{sugita.isamu.sa3,morihi-t,ara,yasumoto\} @is.naist.jp
}

\begin{abstract}
If temporal variation of a person's hunger degree could be estimated, it would be possible to adjust his/her eating habits and/or prevent obesity. It is well-known that there is a negative correlation between a hunger degree and a blood glucose level. However, it is hard to measure a person's blood glucose level anytime and anywhere, because it relies usually on an invasive method (e.g., blood sampling). This paper proposes a method for estimating a person's hunger degree in a non-invasive way. Our proposed method is composed of (1) a blood glucose level estimation model based on logs of meals and exercises, and (2) a hunger degree estimation model based on the estimated glucose level. The former model is constructed by correlating an actual blood glucose level and logs of meals and exercises with a machine learning technique. Here, the actual blood glucose level is measured by a commercial blood glucose meter invasively. The latter model is constructed by associating the measured blood glucose level with a subjective hunger degree. We also design and develop a mobile application for facilitating a user to easily record meals and exercises information. Through an experiment with a subject, we confirmed that our system can estimate a blood glucose level within about $14 \%$ mean percentage error and finally estimate hunger degree within about 1.3 levels mean error among 10 levels.
\end{abstract}

Keywords-hunger degree estimation, blood glucose level estimation, non-invasive method, meal and exercise information, machine learning, mobile application

\section{INTRODUCTION}

Recently, eating habits are becoming worse all over the world, and this fact is causing the increase of obese people. Obesity, a growing epidemic in mature societies, is an excess of body fat that often results in significant impairment of health. Especially in U.S., WHO (World Health Organization) reported that obese people in the world have nearly doubled since 1980, and the 1.4 billion adults are overweight [1]. Because obesity causes various chronic diseases such as heart disease, stroke, diabetes, etc., it is quite important to improve a person's eating habits for preventing obesity and those diseases.

There are many studies to support a user's health and dietary life by estimating his/her health condition, etc. For example, a system observing a balance of a user's caloric intake and expenditure [2], a blood glucose estimation system for diabetic patients [3], and a system recommending a suitable dietary intake for assisted-living resident [4] have already been developed.

Eating at inappropriate time and eating too much are typical bad eating habits. Such bad eating habits may be caused by the fact that a person is not always aware of his/her hunger degree (intensity of hunger). Therefore, if a person's hunger degree could be estimated, it would be possible to inform him/her of appropriate time and amount of a meal for improving his/her eating habits. An internal state of a human like a hunger degree can be measured by a special instrument, although such an instrument is expensive and hard to carry. Moreover, the special instruments used in [2] and [3] require an invasive way to measure the user's internal state. Therefore, it is not suitable to use it daily and continually. In order to prevent epidemic obese effectively, an easy and non-invasive method for estimating hunger degree is desired.

In this paper, we propose a non-invasive method for estimating a user's hunger degree from a log of meals and exercises which can easily be recorded through a smartphone application. We compose the proposed method of two estimation models. The first model is to estimate a blood glucose level from a log of taken meals and exercises. The reason why we first estimate glucose level is that it has a strong correlation with a hunger degree [5]. The second model is to estimate a hunger degree from the blood glucose level estimated by the first model. To construct the blood glucose level estimation model, we measure the actual blood glucose level by a commercial blood glucose meter invasively, and associate the measured data with the log of meals and exercises by a machine learning technique. A blood glucose level has a feature that it quickly increases after meal, and slowly decreases and converges asymptotically to the lowest level. Therefore, we employ a log-normal distribution as a fitting function. To construct a hunger degree estimation model, we associate the estimated blood glucose level with the subjective hunger degree represented in several levels, taking it into account that a hunger degree quickly decreases after meal, slowly increases up to the peak level, and varies earlier than a blood glucose level.

As an evaluation experiment, daily blood glucose level were measured and a $\log$ of daily meals and exercises was recorded for a subject, and we applied the proposed method to the measured/recorded data. As a result, we confirmed that our method can estimate a blood glucose level within about $14 \%$ mean percentage error and finally estimate hunger degree within about 1.3 levels mean error among 10 levels.

\section{RELATED WORK}

Lester et al. [2] proposed a system for automatically estimating caloric expenditure in monitoring a balance between energy intake and expenditure. Acceleration and a moving distance during exercise and the densities of oxygen and carbon dioxide included in aspiration and intake air are used 
to estimate caloric expenditure. In this method, subjects must wear acceleration, oxygen, and carbon dioxide sensors while performing a set of indoor and outdoor exercises. The sensor data are then applied to an estimation model to obtain the estimated caloric expenditure. Although this study estimates physiological states, it focuses on estimating caloric expenditure and requires dedicated and exaggerated sensors to measure oxygen and carbon dioxide levels from breath.

Yamaguchi et al. [3] estimates blood glucose level based on data mining techniques from the data measured by a metabolic rate monitor and a blood glucose monitor. An application software calculates caloric intake from meal information. The proposed blood glucose level estimation model achieves accuracy of $90 \%$. However, this method can estimate the blood glucose level only in the morning and requires a user (targeting diabetes patient) to measure his/her blood glucose level everyday. This method also requires multiple devices (i.e., a PC and a metabolic rate monitor), so it is costly.

De Pessemier et al. [6] proposed a method which estimates blood glucose levels and systolic and diastolic blood pressure levels in a non-invasive way using a photoplethysmograph (PPG) and machine learning techniques. This method estimates these levels by applying a machine learning algorithm to the data obtained from a PPG sensor, an activity detection module, and a signal processing module. Through an experiment to apply the method to 410 subjects, it was confirmed that $87.7 \%$ of estimations were clinically accurate. However, this method requires special and costly instruments to obtain data for estimation.

Ciampolini et al. [7] classified a subjects group which have disease of the gastrointestinal system into the training group and the test group, and showed that the blood glucose level of a test group subject can be estimated within about 14-16\% error from his/her intensity of hunger feeling based on the learning result (i.e., correlation between intensity and blood glucose level) in the training group. This method estimates the blood glucose level from the subjective hunger degree, that is a reverse of our method. Moreover, this study relies on the subjective feeling of subjects.

\section{Challenges to Hunger Degree Estimation AND BASIC IDEAS FOR SOLUTION}

In this section, we describe the target environment and the information of a user required for estimating hunger degree, then clarify the challenges to be tackled.

\section{A. The target environment/users and the required user infor- mation}

We target ordinary users with the following properties.

- The user who usually takes three meals a day, or at least one meal a day.

- The user who is healthy and whose blood glucose level is within a normal range (i.e., the user does not have diabetes).

- The user who usually performs exercise like walking.

- The user who usually uses a mobile terminal such as a smart-phone and a tablet which can record a log of meal and exercise.
TABLE I. ITEMS INCLUDED IN MEAL INFORMATION

\begin{tabular}{c||c}
\hline \multicolumn{1}{l||}{ Item } & Content \\
\hline Time & The time of taking meal \\
\hline Amount & Perceived quantity level of meal (10 levels) \\
\hline Fulfillment & Feeling of fullness after meal (10 levels) \\
\hline
\end{tabular}

TABLE II. ITEMS INCLUDED IN EXERCISE INFORMATION

\begin{tabular}{c||c}
\hline \multicolumn{1}{c||}{ Item } & Content \\
\hline Number of steps & Total number of steps in the day \\
\hline Time & The time when the number of steps was recorded \\
\hline Amount of sleep & $\begin{array}{l}\text { Existence of sleeping hours } \\
(0 \text { or } 1) \text { after the previous } \\
\text { record and the length of sleep- } \\
\text { ing hours }\end{array}$ \\
\hline Caloric expenditure & Caloric expenditure in the day \\
\hline
\end{tabular}

The meal information consists of the items shown in Table $\mathrm{I}$, and is recorded after each meal (breakfast, lunch, and dinner) and each between-meal eating. Since it can be a burden for a user to input the amount of meal precisely, the amount as well as the feeling of fullness are roughly specified in 10 levels.

As shown in Table II, the number of steps, the time of exercise, existence/length of sleep hours, and caloric expenditure in the current day are recorded as exercise information. Most of these information can be automatically measured by using dedicated smart-phone applications.

\section{B. Challenges and basic ideas for solution}

The following two challenges are considered for estimating the hunger degree using our method.

1) It is difficult to quantify the hunger degree, because it is a sensory quantity.

2) It is difficult to achieve high estimation accuracy when the input information is scarce or ambiguous.

The first challenge is due to the fact that there are no quantitative indices that capture the sensory state such as hunger degree and fatigue degree. One approach to estimate these degrees are to observe a slight change in vital signals (e.g., body temperature, heart rate, etc.) caused by the change in the sensory state. Some existing studies use dedicated measurement devices to capture such a slight change in vital signals. In these studies, however, a user is required to wear special devices and/or to accept invasive measurements. Unlike the existing studies, we employ the following approaches to estimate the user's hunger degree.

- We focus on the blood glucose level, which is closely related to the hunger degree, and estimate the temporal variation of the blood glucose level from a log of meals and exercises which can be recorded by a mobile terminal.

- We estimate the hunger degree from the estimated blood glucose level by correlating the two values with each other.

In the above approach, estimation models (for glucose level and hunger degree) for each pre-defined user type must be constructed through invasive measurements of the blood glucose levels of representative users who belong to the corresponding type. After the model construction, the blood glucose level 
of a general user can be estimated by specifying the type of the user and using the pre-constructed models without invasive measurements.

More details of the above approach are given in Sect. IV.

The second challenge is caused by the fact that the human's sensory state like hunger degree is rather vague and its intensity changes depending on the various external factors. Therefore, to precisely estimate the hunger degree, it is required to obtain detailed and precise input information about meal and exercise. In our target environment, the estimation error would increase because the input information is simplified to facilitate a user to easily input meal information (amounts and a feeling of fullness) by just selecting one among 10 levels. Nevertheless, in our method, we target only healthy users, thus the required accuracy of blood glucose level estimation is not always high compared with the case when targeting diabetes patients.

\section{Hunger Degree Estimation Method}

The proposed hunger degree estimation method is composed of two models: a model for estimating a blood glucose level from a $\log$ of meals and exercises and a model for estimating a hunger degree from the estimated blood glucose level. In this section, we describe how we construct these models.

\section{A. Blood glucose estimation from meal and exercise logs}

Blood glucose level is an index about a concentration of glucose in a blood, and this value is about $70 \sim 109[\mathrm{mg} / \mathrm{dL}]$ in a hungry state, and is lower than $140[\mathrm{mg} / \mathrm{dL}]$ two hours after meal [3]. Blood glucose level reaches a peak in some time interval after meal, and declines gradually. To estimate a blood glucose level, we employ a log-normal distribution function shown in formula (1) as a fitting function since it has a similar curve to the blood glucose transition. In the formula, $x$ denotes a variable, $\mu$ an average, and $\sigma$ a standard deviation.

$$
f(x, \mu, \sigma)=\left\{\begin{array}{l}
\frac{1}{\sqrt{2 \pi} \sigma x} \exp \left\{\frac{-(\log x-\mu)^{2}}{2 \sigma^{2}}\right\}, \quad x>0 \\
0, \quad x \leq 0
\end{array}\right.
$$

In our proposed method, a variable of the log-normal distribution function corresponds to the current time, $t$. In addition, the average $\mu$ and the standard deviation $\sigma$ correspond to parameters of the estimation model which depend on the individual difference of the blood glucose transition. The blood glucose estimation function $G(t)$ is shown in equation (2). Here, $t$ and $t_{0}$ denote the current time and the time of the previous estimation, respectively, $w_{i}$ and $w_{e}$ denote weight coefficients of meal and exercise information, respectively, $c_{g}$ denotes a magnification coefficient, $I(t)$ and $E(t)$ denote normalized amounts of meals and exercises taken during $\left[t_{0}, t\right]$.

$$
G(t)=c_{g}\left[w_{i} I(t) f\left(t-t_{0}, \mu, \sigma\right)-w_{e} E(t)\right]+G\left(t_{0}\right)
$$

The blood glucose estimation function $G(t)$ is defined as a log-normal distribution function and values of parameters $c_{g}, w_{i}, w_{e}, \mu, \sigma$ used in $G(t)$ must be optimized to minimize

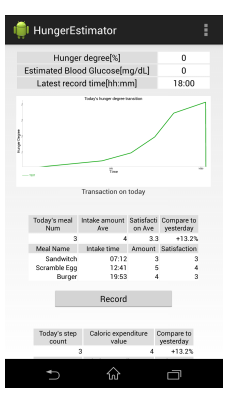

(a)

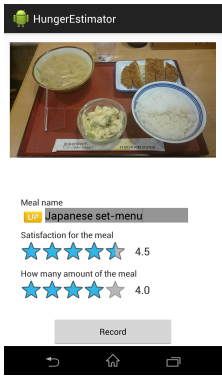

(b)

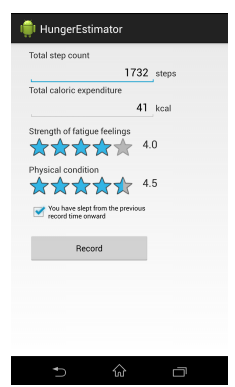

(c)
Fig. 1. Example of Smart-Phone Application Interface

the estimation error. The semi-optimal values of these parameters are obtained by fitting to the equation (2) some training data samples which are collected in the preliminary experiment. Here, the least square method is used for the fitting process.

\section{B. Hunger Degree Estimation from Blood Glucose Level}

A model for estimating the hunger degree from the estimated blood glucose level is shown in equation (3).

$$
H(t)=l_{\max } \cdot\left(1-\frac{G\left(t-T_{g}\right)}{g_{\max }}\right)
$$

Here, $l_{\max }$ is a constant value (e.g., 10) representing the maximum level of hunger degree, and $T_{g}$ is a constant time interval for hastening glucose level transition, and $g_{\max }$ is the maximum glucose level for normalizing the glucose level.

The hunger degree transition gets behind the glucose level transition by some time interval (e.g., 90 minutes). Thus, in equation (3), parameter $T_{g}$ is used and the optimal value of $T_{g}$ for estimation is obtained through fitting some samples to the equation.

\section{SMART-PHONE APPLICATION}

We suppose to implement the proposed method as an Android application which aims to allow users to easily input meal and exercise information and obtain a feedback (i.e., blood glucose level and hunger degree).

We show an overview of the proposed smart-phone application in Fig. 1. Fig. 1 (a) is a main interface which allows a user to confirm a temporal variation of the estimated blood glucose level and hunger degree within the day until the present time, as well as the logs of meal and exercise. The interface shown in Fig. 1 (b) is for inputting meal information: a user can register a picture and a name/type/amount of a meal as well as a feeling of fullness after the meal (in 10 levels). The interface shown in Fig. 1 (c) is for confirming exercise information, that is, foot steps, calories expenditure, etc. which are obtained from other applications. A degree of fatigue, physical condition, sleeping time, etc can also be input via this interface. Through this application, a user can easily input meal and exercise information and obtain temporal variation of estimated blood glucose level and hunger degree. 


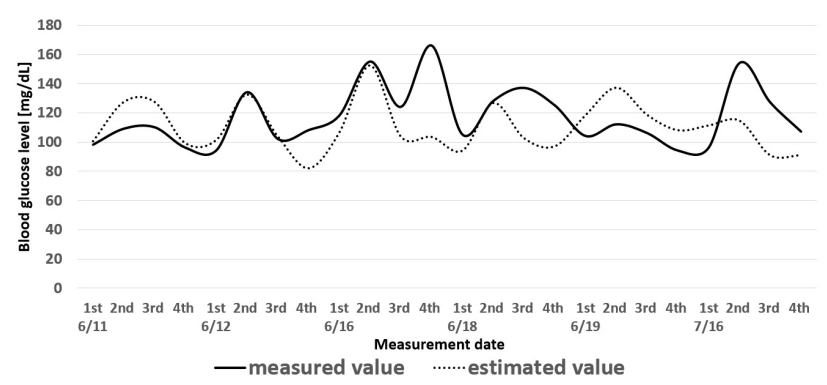

Fig. 2. The transition of the measured and estimated blood glucose level

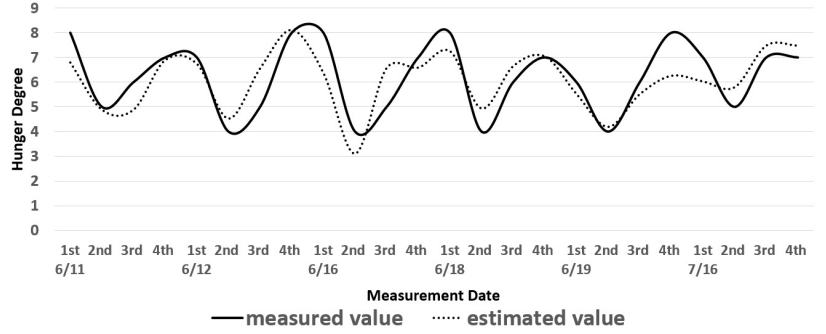

Fig. 3. The transition of the answered and the estimated hunger degree

\section{EXPERIMENT}

Experimental method: The purpose of the experiment is to confirm the estimated blood glucose level and the corresponding hunger degree which our models estimate. For this purpose, we asked a subject (male, 20's) to record logs of meals and exercises and measure the blood glucose level for 8 days.

We used a blood glucose meter GLUCOCARD G Black [8] with Self-Monitoring Glucose Kit G-Sensor [9] and puncture devices [10] of Arkray. The blood glucose meter can measure the blood glucose level by puncturing a finger and drawing a small amount of blood. In the experiment, the blood glucose level was measured four times a day depending on the time of logging meals and exercises.

The subject input the following meal and exercise information in an Excel sheet prepared in advance.

- $\quad$ time and subjective hunger degree in 10 levels (this information is used as a ground truth of the hunger degree)

- time and amount (in 10 levels) of each meal and each between-meals eating

Estimation result of blood glucose level: Fig. 2 shows temporal variation of measured and estimated blood glucose level, where the solid line and the dashed line correspond to the measured and the estimated, respectively. Fig. 2 suggests that our estimation reproduces up-and-down timings accurately. The mean percentage error (MPE) of our estimation is about $14 \%$ and the correlation coefficient is about 0.30. However, the size of variation between the measured and the estimated is largely different in some places. We confirmed that such a large difference is caused by some special meal type (e.g., the size of variation is much smaller when eating Udon than eating rice). Furthermore, our present estimation models need to be extended to adapt to various users with different physical features or different ages. As future work, we plan to extend our estimation models by reflecting meal type and individual difference.

Estimation result of hunger degree: We show the result of hunger degree estimation in Fig. 3. The result shows that our model for estimating hunger degree can accurately estimate the hunger degree in both time and the size of variation. The mean error is about 1.3 of 10 levels and the correlation coefficient is about 0.77 .

\section{CONCLUSION}

In this paper, we proposed a method for estimating hunger degree of a user from logs of meal and exercise information of the user, which consists of two steps: blood glucose level estimation from logs and hunger degree estimation from the blood glucose level. We also implemented a smart-phone application which allows a user to easily input the information of meal and exercise. We conducted an experiment where we collected $\operatorname{logs}$ of meal and exercise information of a subject and applied our method to the collected logs. As a result, we confirmed that our method can estimate the blood glucose level with about $14 \%$ mean percentage error and the hunger degree with about 1.3 levels mean error among 10 levels. For future work, we plan to improve the accuracy of our estimation models by reflecting meal type, develop applications utilizing hunger degree estimation, and explore the possibility to adapt our estimation models to individual difference.

\section{ACKNOWLEDGMENT}

This work is partially supported by SCOPE (Strategic Information and Communications R\&D Promotion Programme).

\section{REFERENCES}

[1] W. H. Organization., "Obesity and overweight," http://www.who.int/mediacentre/factsheets/fs311/en/.

[2] J. Lester, C. Hartung, L. Pina, R. Libby, G. Borriello, and G. Duncan, "Validated caloric expenditure estimation using a single body-worn sensor," in Proceedings of the 11th international conference on Ubiquitous computing. ACM, 2009, pp. 225-234.

[3] M. Yamaguchi, S. Kanbe, K. Wårdell, K. Yamazaki, M. Kobayashi, N. Honda, H. Tsutsui, and C. Kaseda, "Trend estimation of blood glucose level fluctuations based on data mining," in The 7th world multiconference on systemics, cybernetics and informatics, 2003, pp. 86-91.

[4] T. De Pessemier, S. Dooms, and L. Martens, "A food recommender for patients in a care facility," in Proceedings of the 7th ACM Conference on Recommender Systems, ser. RecSys '13. New York, NY, USA: ACM, 2013, pp. 209-212. [Online]. Available: http://doi.acm.org/10.1145/2507157.2507198

[5] M. Timothy S. Harlan, "Dr. gourmet : hunger, blood glucose, and the glycemic index," http://www.drgourmet.com/bites/2008/031208.shtml.

[6] E. Monte-Moreno, "Non-invasive estimate of blood glucose and blood pressure from a photoplethysmograph by means of machine learning techniques," vol. 53, pp. 127-138.

[7] M. Ciampolini and R. Bianchi, "Training to estimate blood glucose and to form associations with initial hunger," vol. 3, no. 42, 2006.

[8] Arkray, "Gluco card g black," http://www.arkray.co.jp/press/press/2013_ 05_07.html.

[9] — - "G sensor," https://biz.arkray.co.jp/BunsyoKaitei/Gsensor.pdf.

[10] —, "Naturalet ez device," https://biz.arkray.co.jp/BunsyoKaitei/ naturaletEZ.pdf. 